\title{
LAS ENCUESTAS DE OPINIÓN PÚBLICA EN AMÉRICA LATINA: UNA MIRADA DESDE LOS PROPIOS ENCUESTADORES
}

\author{
María Braun ${ }^{1}$
}

\section{A modo de introducción}

Las encuestas de opinión pública son, sin ninguna duda, un campo controversial. Desde las afirmaciones -no siempre evidentes- de que impactan en el comportamiento electoral de los ciudadanos hasta las descalificaciones de quienes afirman no creer en ellas, pasando por las sospechas de manipulación de los datos o las críticas a sus errores en las predicciones electorales, lo cierto es que su relevancia y su protagonismo en épocas preelectorales se ve muchas veces enturbiado por estos cuestionamientos.

Quizás el marco teórico más útil y conocido para explicar esta particularidad de las encuestas sea el de Dominique Wolton (1995). Para este teórico de la comunicación, las encuestas se instalan en un campo -el de la comunicación política- donde participan e intercambian discursos tres actores diferentes: los políticos, los periodistas y la opinión pública (que se expresa, sobre todo, a través de los sondeos). En ese espacio, las encuestas -pese a ocupar un papel relevante especialmente en épocas de eleccionesno se mueven con independencia de los políticos que las encargan ni de los medios que las difunden. Eso las coloca,

Socióloga, Universidad de Buenos Aires (UBA) y Magíster en Desarrollo Urbano, University College London (UCL). Socia fundadora y presidente de MBC MORI y presidente de WAPOR Latinoamérica. 
entonces, en un campo controversial donde, además, su capacidad para influir es obviamente mucho menor que la de los políticos o la de los medios de comunicación.

Conocer la mirada de los profesionales de las encuestas sobre su propia actividad parece un dato relevante, más aún si se tiene en cuenta que estamos frente a un campo que, si bien viene experimentando un crecimiento importante, no tiene muchos más años en América Latina que los que tiene el retorno a la democracia.

Con este objetivo -el de describir el lugar que los propios encuestadores le otorgan a su actividad, conocer la imagen que tienen de ella así como la que le atribuyen a otros sectores, como las élites y la opinión pública- es que en 2010, conjuntamente con Luis Eduardo González, hicimos desde WAPOR Latinoamérica una encuesta a profesionales de las encuestas (encuestadores y académicos). La idea general del estudio fue hacer un relevamiento básico de la situación de la profesión y de las críticas que recibe, de modo tal de contar con información que contribuya a profesionalizar la actividad y a generar mecanismos de autorregulación.

Los principales resultados de esta encuesta-que fueron publicados en el número 1 de la Revista Latinoamericana de Opinión Pública y están accesibles en http://www.waporlatinoamerica.org/descargas/documentos/Maria_Braun. pdf- muestran que, pese a ser una actividad que, en la opinión de los expertos que la llevan a cabo, responde a los estándares internacionales, resulta fuertemente cuestionada, tanto interna como externamente. Siempre según los encuestadores, las críticas a las encuestas son muy frecuentes y provienen de diferentes sectores -predominantemente de los medios, pero también de los partidos políticos, de los gobiernos y, en menor medida, de los medios académicos-. La principal crítica que reciben las 
encuestas es la de falsear o esconder resultados por razones partidistas o económicas.

Cuatro años después, parece necesario actualizar esta información y darle mayor robustez a los datos. En ambos casos -tanto en 2010 como en 2014- se utilizó la misma metodología para el relevamiento de los datos (encuesta on line) y un diseño de muestra similar: partiendo de la lista de miembros latinoamericanos de WAPOR, se utilizó la técnica de "bola de nieve": se le pidió a referentes clave de la actividad que sugirieran nombres de colegas. Si en 2010 la encuesta sumó 139 entrevistas, en marzo de 2014, cuando se hizo el campo de esta segunda medición, se realizaron 231 entrevistas en los mismos países que en el estudio anterior (Argentina, Brasil, Chile, Colombia, Costa Rica, Ecuador, Honduras, México, Paraguay, Perú, República Dominica, Uruguay y Venezuela). Por otro lado la muestra incluyó, además de a encuestadores y a académicos ligados a las encuestas, a periodistas familiarizados con el uso de los datos de aquellas.

Siempre en relación con las características de la muestra, es importante señalar que, por razones que seguramente tienen que ver con el nivel de inserción y el trabajo de WAPOR en estos países, sólo en Argentina y en Uruguay se lograron tamaños de muestras que cubrieron más que razonablemente el universo considerado. En el resto de los países las muestras son pequeñas, particularmente en el caso de Brasil, donde la actividad tiene un nivel de desarrollo muy importante. De ahí que en los datos que mostramos en este trabajo sólo las respuestas relativas a Argentina y a Uruguay aparecen identificadas; para el resto de los países incluidos en el estudio, los datos se presentan abiertos por país pero no identificables. 


\section{Gráfico 1: País de residencia ${ }^{2}$}

\begin{tabular}{|l|c|c|}
\hline & $\mathbf{n}=$ & $\%$ \\
\hline País de residencia & 64 & $29 \%$ \\
\hline Argentina & 26 & $11 \%$ \\
\hline México & 23 & $10 \%$ \\
\hline Uruguay & 22 & $10 \%$ \\
\hline Chile & 17 & $7 \%$ \\
\hline Colombia & 15 & $6 \%$ \\
\hline Brasil & 13 & $6 \%$ \\
\hline Ctro. Am y Caribe & 12 & $5 \%$ \\
\hline Bolivia & 12 & $5 \%$ \\
\hline Perú & 10 & $4 \%$ \\
\hline Ecuador & 7 & $3 \%$ \\
\hline Venezuela & 10 & $4 \%$ \\
\hline Otros & $\mathbf{2 3 1}$ & $\mathbf{1 0 0} \%$ \\
\hline Total & & \\
\hline
\end{tabular}

Total entrevistados ( $n=231$ ) Fuente: WAPOR Latinoamérica

Abril - Mayo, 2014 - Pág. 1

Como datos adicionales y de interés en relación con la muestra, cabe señalar que más de la mitad de los entrevistados (el 56\%) declara trabajar en una firma encuestadora o consultora, mientras que el $29 \%$ lo hace en la academia y el $16 \%$ trabaja como periodista. Por último, casi dos tercios de los entrevistados (el 64\%) tienen estudios de posgrado, en gran parte de los casos realizados en el exterior.

En todos los casos, y teniendo en cuenta que varios de los profesionales entrevistados operan en diferentes países, se les pidió a todos que respondieran a la encuesta refiriéndose a su país de residencia. 


\section{Sobre la imagen de las encuestas y de los encuestadores}

Según los encuestadores, poco menos de la mitad del público informado considera que las empresas encuestadoras más conocidas trabajan seriamente. Estas opiniones, que se registran en todos los subgrupos estudiados sin importar que se trate de encuestadores, académicos o periodistas, ni cuál sea la formación académica del entrevistado, varían en cambio fuertemente según país. Así, mientras que en Uruguay la mayoría de los entrevistados considera que la imagen de las encuestas entre el público informado es positiva, en la Argentina este porcentaje disminuye a un $20 \%$. 
Gráfico 2: Imagen de las encuestadoras conocidas entre el público informado según país

Entre el público informado, ¿cuál es la actitud predominante respecto a la seriedad profesional de las encuestas de opinión pública política de las encuestadoras más conocidas?

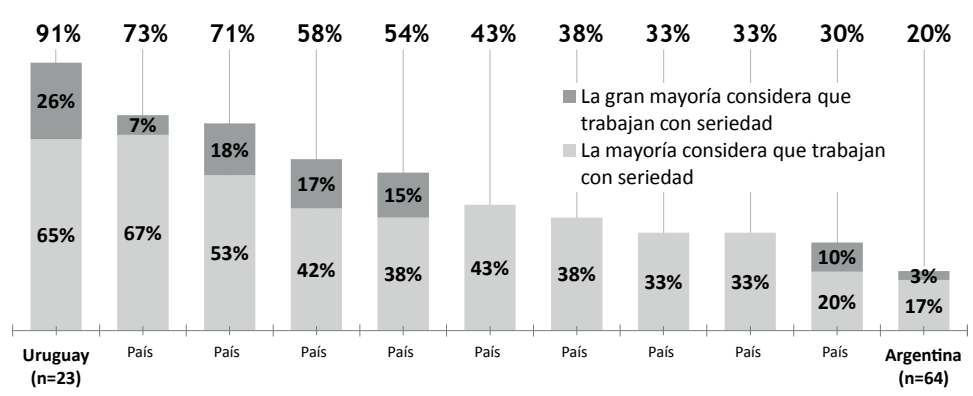

Total entrevistados $(n=231)$

Fuente: WAPOR Latinoamérica

Abril - Mayo, 2014 - Pág. 2

Algo similar ocurre en relación con la imagen de las encuestas y de las empresas encuestadoras entre el púbico general. Aquí también las diferencias según país son muy grandes -aunque algo menores que entre el público informado-: Uruguay es el país donde es mejor la imagen que la opinión pública tiene de las encuestas y Argentina está entre los que presentan porcentajes bajos de imagen positiva. 
Gráfico 3: Imagen de los encuestadores y de las empresas encuestadoras entre el público general (total América Latina y por país)

¿Qué imagen cree Ud. que tiene la opinión pública, los ciudadanos, de los encuestadores y las empresas encuestadoras?

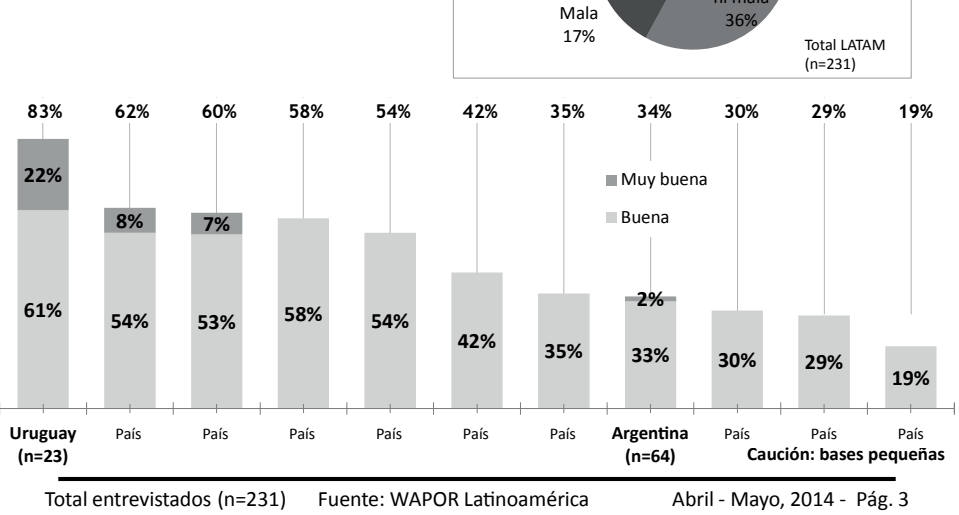

Según más de dos tercios de los entrevistados (el 69\%) las críticas a las encuestas son muy frecuentes y provienen, fundamentalmente, de los medios de comunicación y de los partidos políticos y el gobierno, aunque también de la academia. De la comparación con los datos de 2010 surgen diferencias poco significativas, lo que nos estaría mostrando que el escenario no se ha modificado en los últimos años: a las encuestas se les reprocha falsear o manipular los datos, sea por partidismo político o por razones económicas. También se les cuestiona una capacidad excesiva para imponer agenda y para influir en el voto. 


\section{Gráfico 4: Críticos más frecuentes de las encuestas (América Latina, 2010 y 2014)}

\section{¿Quiénes son los críticos más frecuentes de las encuestas? \\ RESPUESTAS MÚLTIPLES}

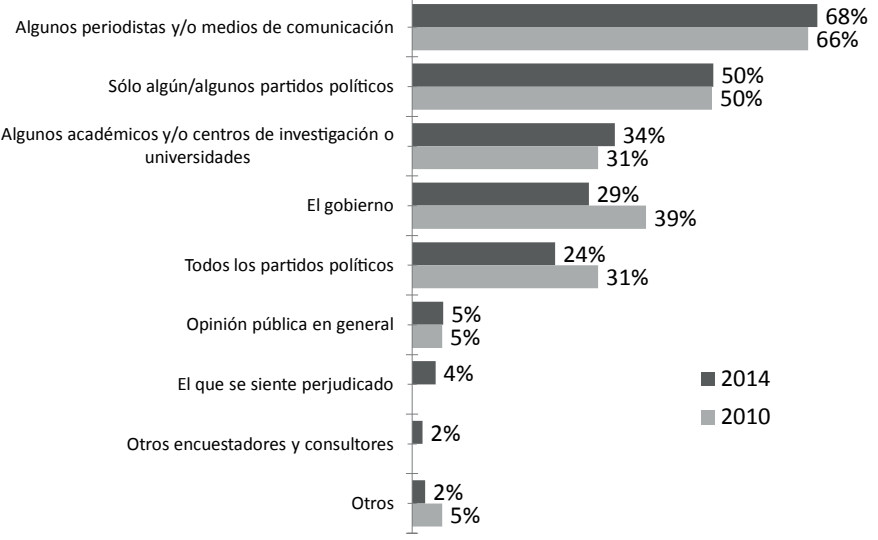

Total entrevistados ( $n=231$ ) Fuente: WAPOR Latinoamérica

Abril - Mayo, 2014 - Pág. 4 


\section{Gráfico 5: Principales críticas a las encuestas}

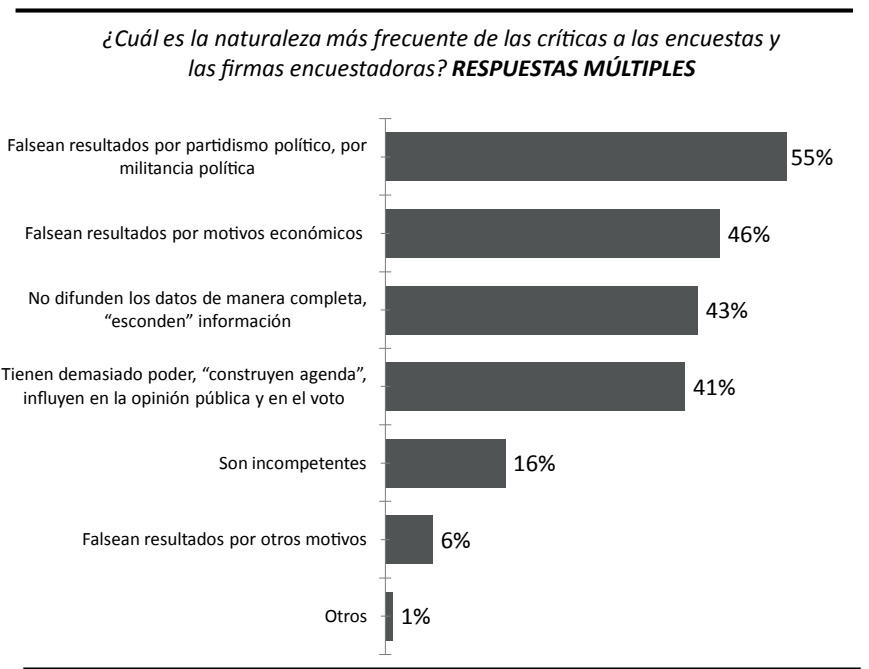

Total entrevistados $(n=231) \quad$ Fuente: WAPOR Latinoamérica

Abril - Mayo, 2014 - Pág. 5

No se cuestiona, en cambio, la calidad de las encuestas: pese a ciertas limitaciones de tipo técnico o económico, la gran mayoría de los encuestados les atribuye una calidad razonable y comparable a la de los países con democracias consolidadas. En este sentido, cabe señalar que si bien se observan diferencias por país, éstas son mucho menos significativas que las diferencias que se observan según la imagen de las encuestas. 


\section{Gráfico 6: Percepción de la calidad de las encuestas en América Latina}

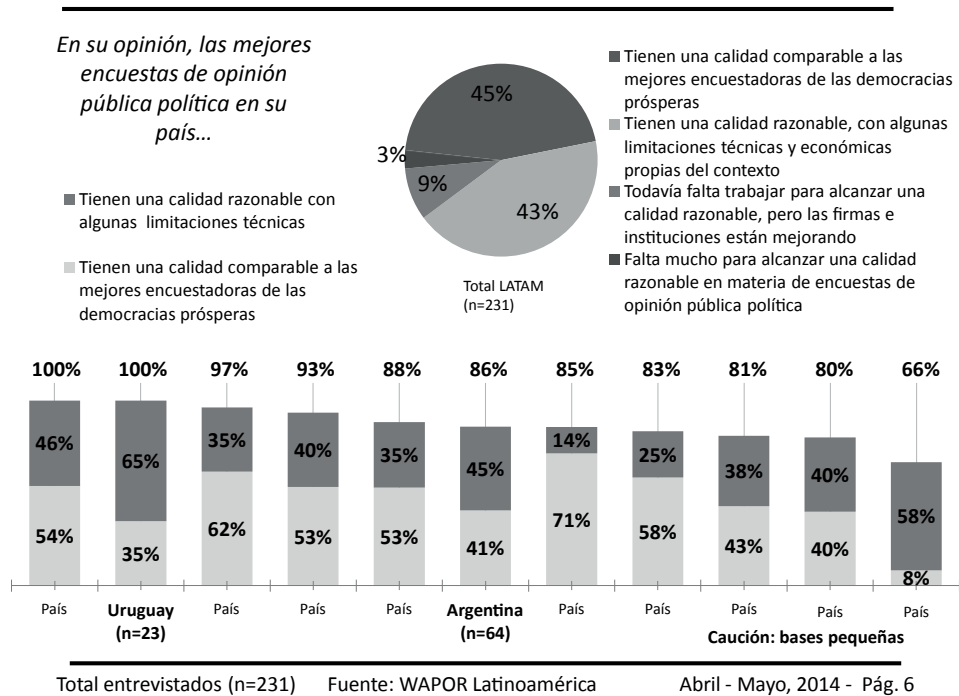

\section{Sobre la publicación de las encuestas}

No existe un estándar latinoamericano en relación con la publicación de las encuestas. Así, en algunos países -entre los que se encuentra Uruguay- la gran mayoría de los entrevistados (el 79\%) considera que la calidad de la publicación de las encuestas es muy buena o razonablemente buena. En otros países estos porcentajes disminuyen al $40 \mathrm{o}$ al 32\%, y Argentina se ubica en un valor intermedio (60\%). 
Gráfico 7: Evaluación de la calidad de la publicación de las encuestas según país

Cómo evalúa Ud. la calidad de la publicación de las encuestas en los principales medios del país?

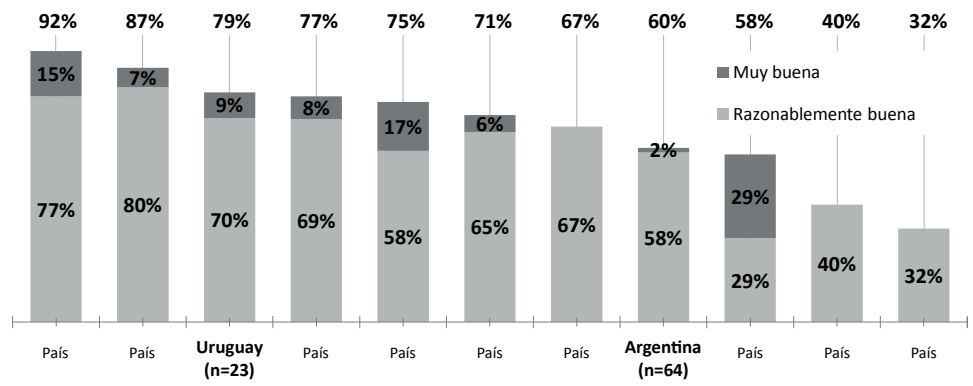

Caución: bases pequeñas

El dato que presentamos a continuación refuerza la idea de que los datos de las encuestas que llegan a la opinión pública no son siempre, según los propios encuestadores, "buenos". En efecto, cuando preguntamos cuántas son las firmas encuestadoras que difunden sus datos en los medios, el promedio para América Latina es de 5,61 firmas. Y cuando preguntamos cuántas de esas firmas e instituciones que difunden encuestas realizan un trabajo profesional de buena calidad ese promedio de menciones disminuye a 3,23. En síntesis, los medios publican datos que no siempre son confiables. 
Gráfico 8: Sobre la difusión y la calidad de los datos publicados (promedios), América Latina

Preg: "Al margen de su reputación, ¿cuántas firmas y/o instituciones difunden regularmente encuestas de opinión pública política (intención de voto nacional, evaluación de la gestión del gobierno, otros temas políticos) en medios de comunicación masiva?" (Marcar número total de firmas que difunden encuestas)

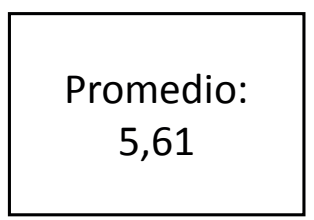

Preg.: “¿Cuántas de esas firmas e instituciones que difunden encuestas diría Ud. que realizan un trabajo profesional de buena calidad?" (Marcar número de firmas que realizan encuestas de buena calidad)

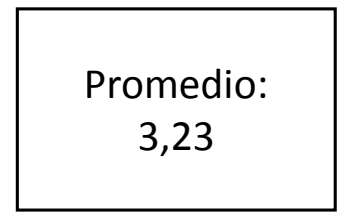

Otra vez, las diferencias según país son muy significativas y la brecha entre la cantidad de empresas cuyos datos se difunden regularmente en los medios y las que realizan un trabajo de buena calidad varía fuertemente según país. Argentina es el país donde esta brecha es más alta; Uruguay otra vez se ubica en uno de los valores más bajos. 


\section{Gráfico 9: Sobre la difusión y la calidad de los datos publicados (promedios), según país}

Al margen de su reputación, ¿cuántas firmas y/o instituciones difunden regularmente encuestas de opinión pública política (intención de voto nacional, evaluación de la gestión del gobierno, otros temas políticos) en medios de comunicación masiva?

¿Cuántas de esas firmas e instituciones que difunden encuestas diría Ud. que realizan un trabajo profesional de buena calidad?

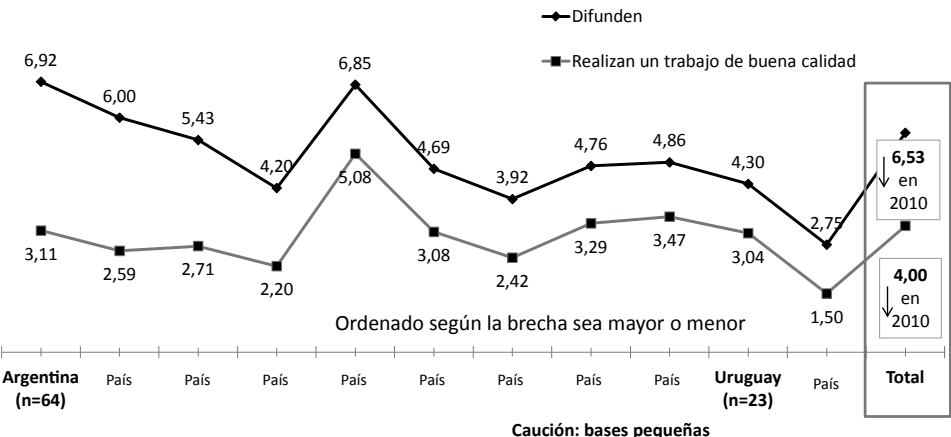

Caución: bases pequeñas

La idea de que la calidad y la credibilidad de las encuestas mejorarían si cada medio tuviera su propia encuestadora, no tiene mayor peso entre los encuestadores, y la mayoría (el 68\%) considera que es mejor que los medios publiquen los datos de todas las encuestadoras. En todo caso, sí se menciona de manera espontánea la necesidad de que los medios profesionalicen a sus periodistas, formándolos en el análisis de los resultados de las encuestas. 
Gráfico 10: La mejor relación entre las encuestadoras y los medios (total América Latina y por país)

¿Pensando en la calidad y en la credibilidad de las encuestas, qué piensa Ud. que es mejor?

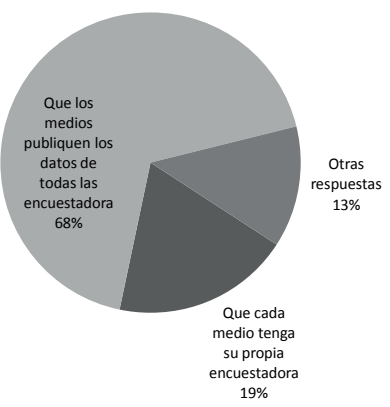

\begin{tabular}{|c|c|}
\hline & $\begin{array}{l}\text { Que cada medio } \\
\text { tenga su propia } \\
\text { encuestadora }\end{array}$ \\
\hline Ctro. Am y Caribe $(n=13)$ & $38 \%$ \\
\hline México $(n=26)$ & $35 \%$ \\
\hline Argentina $(n=64)$ & $27 \%$ \\
\hline Colombia $(n=17)$ & $18 \%$ \\
\hline Bolivia (n=12) & $17 \%$ \\
\hline Venezuela $(n=7)$ & $14 \%$ \\
\hline Ecuador $(n=10)$ & $10 \%$ \\
\hline Chile $(n=22)$ & $10 \%$ \\
\hline Brasil $(n=15)$ & $7 \%$ \\
\hline Uruguay $(n=23)$ & $4 \%$ \\
\hline Perú $(n=12)$ & - \\
\hline
\end{tabular}

Total entrevistados ( $\mathrm{n}=231$ ) Fuente: WAPOR Latinoamérica

Abril - Mayo, 2014 - Pág. 10

Por último, y siempre en relación con la cuestión de la publicación de las encuestas, cabe señalar que, si bien la mayoría de los encuestadores están al tanto de que existen restricciones para la publicación de los datos en épocas previas a las elecciones, el nivel de conocimiento respecto de cómo son esas restricciones y de cuántos días previos a las elecciones incluyen es bastante bajo.

\section{Para terminar}

Por un lado, el estudio ratifica las conclusiones obtenidas hace cuatro años: las encuestas no tienen, según los encuestadores, una buena imagen entre el público 
informado ni en la opinión pública. Y lo que es más importante: los propios encuestadores consideran que en muchos casos los datos que se presentan a la opinión pública son poco confiables.

Pero, además, el estudio aporta un dato que me parece relevante: las diferencias que existen por país, con dos casos que aparecen como extremos: Argentina y Uruguay. Esto nos sugiere que, además de las características intrínsecas a la actividad, muy bien descriptas por Wolton, y que explican una parte importante de esta imagen controvertida de las encuestas, también tienen un peso muy relevante, seguramente, la forma en que se fue conformando el campo de la actividad en cada uno de los países considerados esto es, la historia de los estudios de opinión pública- así como el lugar que hoy ocupan los estudios de opinión pública en relación con la investigación de mercado, con la investigación académica, con los medios comunicación o con la generación y difusión de datos estadísticos. No es de extrañar que en un país como Argentina, donde desde hace ocho años las estadísticas oficiales son sospechadas de manipulación, ${ }^{3}$ las encuestas de opinión tengan tan poca credibilidad: no la tienen entre las élites, ni entre la opinión pública, ni tampoco, como lo muestran estos datos, entre quienes ejercen la profesión.

En enero de 2007, en un contexto inflacionario innegable, la Dirección del Índice de Precios del Instituto Nacional de Estadísticas y Censos (INDEC) fue intervenida por la Secretaría de Comercio, que arbitrariamente consumó un cambio de la metodología de medición de los precios. A partir de ese momento, se observan grandes discrepancias entre las estadísticas oficiales y las privadas de la tasa de inflación. En 2010 un informe de expertos de cinco universidades nacionales fue contundente a favor de la evidencia de una manipulación en las estadísticas públicas. Recientemente se sumaron nuevas discrepancias sobre otras estadísticas oficiales, como es el caso de la tasa de crecimiento del país o del nivel de empleo. 
En 1950 Paul Lazarsfeld, sociólogo austriaco y uno de los fundadores de la sociología empírica, decía: "la gran contribución del psicoanálisis moderno es que nos ha dado un mayor entendimiento sobre lo que nos pasa. La investigación de la opinión pública puede hacer lo mismo por la comunidad siempre y cuando tome más conciencia de sus potencialidades y muestre más entusiasmo por desarrollarlas. Queremos que todos los ciudadanos respeten el tipo de trabajo que hacemos. Una muy buena forma de obtener este respeto es mostrarles que reconocemos nuestros problemas comunes y que podemos contribuir a su clarificación".

Reconocer estos problemas, trabajar sobre ellos y avanzar en la legitimación del campo de las encuestas forman parte de las tareas de la comunidad de pares. Desde esa perspectiva, WAPOR puede tener un papel relevante en Latinoamérica. Al menos eso es lo que piensan los entrevistados. Así, frente a la pregunta "Cree Ud. que, pensando en nuestra actividad como encuestadores o usuarios de las encuestas, la actividad de instituciones como WAPOR en Latinoamérica es muy valiosa, bastante, poco o nada valiosa", la gran mayoría -el 95\%- afirma que es muy o bastante valiosa. 